\title{
Fluid nonlinearities effect on wake oscillator model performance
}

\author{
Victoria Kurushina ${ }^{1,2, \star}$ and Ekaterina Pavlovskaia ${ }^{1, \star \star}$ \\ ${ }^{1}$ Centre for Applied Dynamics Research, School of Engineering, University of Aberdeen, United Kingdom \\ ${ }^{2}$ Department of Transport of Hydrocarbon Resources, Industrial University of Tyumen, Russia
}

\begin{abstract}
Vortex-induced vibrations (VIV) need to be accounted for in the design of marine structures such as risers and umbilicals. If a resonance state of the slender structure develops due to its interaction with the surrounding fluid flow, the consequences can be severe resulting in the accelerated fatigue and structural damage. Wake oscillator models allow to estimate the fluid force acting on the structure without complex and time consuming CFD analysis of the fluid domain. However, contemporary models contain a number of empirical coefficients which are required to be tuned using experimental data. This is often left for the future work with the opened question on how to calibrate a model for a wide range of cases and find out what is working and is not.

The current research is focused on the problem of the best choice of the fluid nonlinearities for the base wake oscillator model [1] in order to improve the accuracy of prediction for the cases with mass ratios around 6.0. The paper investigates six nonlinear damping types for two fluid equations of the base model. The calibration is conducted using the data by Stappenbelt and Lalji [2] for 2 degrees-of-freedom rigid structure for mass ratio 6.54. The conducted analysis shows that predicted in-line and cross-flow displacements are more accurate if modelled separately using different damping types than using only one version of the model. The borders of application for each found option in terms of mass ratio are discussed in this work, and appropriate recommendations are provided.
\end{abstract}

\section{Introduction}

Vortex-induced vibrations (VIVs) is a form of fluid - structure interaction when fluctuations of the fluid forces due to growing vortices lead to increased fatigue and motion of the slender structure. Flexible structures in shear flows are in the main interest for industries, for example, subsea production systems, but the complex VIV phenomenon is still not explored fully for simpler cases of rigid structures with one and two degrees-of-freedom in the uniform flow.

Increased displacement amplitudes of a structure are observed during the so-called lock-in state (or synchronization) - condition of resonance between the fluid and the structure. Lock-in condition is achieved by matching the natural frequency of the structure and the frequency of vortex shedding.

The current work is performed using wake oscillator approach to VIV phenomenon. This method implies a very simplified representation of the fluid around the structure as fluctuations of lift and drag forces only. This allows to reduce computational time, but requires prior calibration and validation work in order to take empirical parameters correctly.

It was proved by Jauvtis and Williamson [3] in 2004 that development of the resonance state in terms of displacement amplitudes varies for rigid structures with 2

\footnotetext{
^e-mail: v.kurushina@abdn.ac.uk

$\star \star$ e-mail: e.pavlovskaia@abdn.ac.uk
}

degrees-of-freedom depending on the mass ratio. The "border" between two types of lock-in peak was identified around mass ratio 6.0. This is why, the region of mass ratios 5.0-7.0 seems particularly important and challenging for modelling.

Previous work towards improving fluid oscillators for VIV problem included the study of different combinations of Van der Pol and Rayleigh equations [4]. The wider range of oscillators and the developed optimisation procedure are given in details in [5]. The focus of these previous works was on "low" mass ratio around 2.0-4.0, which left other cases for future exploration. In this study, the authors consider the most suitable oscillators and corresponding sets of coefficients for 2DOFs rigid structure with mass ratio around 6.0 with the focus on maximum possible accuracy of prediction for in-line and cross-flow displacement amplitudes. The basis for modification is the model presented in [1].

The current paper is structured as follows. Section 1 introduces the basic ideas that allow to narrow the aim of this study. Section 2 contains details of the improved model and the list of applied fluid nonlinearities of wake equations. Section 3 gives the summary of calibration procedure and presents three groups of calibration results. Section 4 observes advantages and drawbacks of the achieved precision of displacement prediction. And Section 5 gives concluding remarks and perspectives of the future studies. 


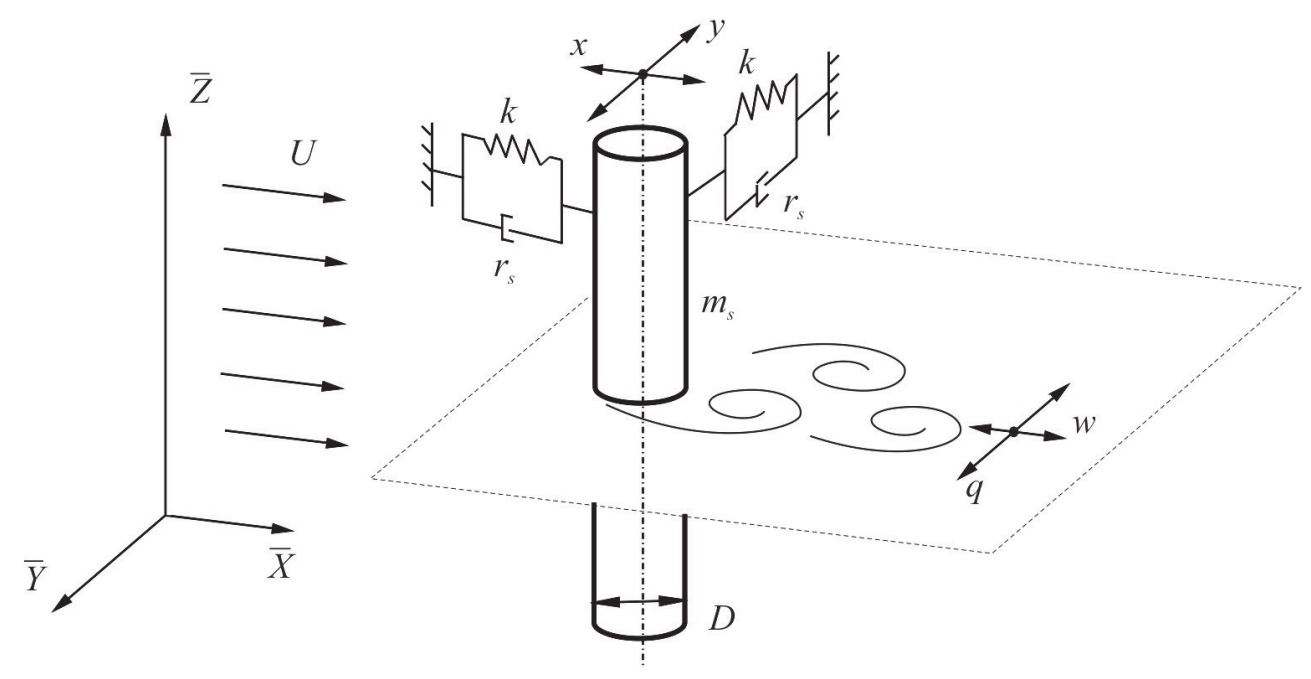

Figure 1. The model of two degrees-of-freedom elastically-supported rigid structure interacting with the uniform flow of velocity $U$ [5]. Here, $m_{s}$ is structural mass, $r_{s}$ is structural damping, and $K$ is stiffness of support.

\section{Fluid nonlinearities}

The considered case is 2 degrees-of-freedom rigid structure shown in Fig. 1, and the flow is assumed to be uniform. The structure can move in the direction of the flow (or in-line direction), and the direction perpendicular to the flow (or cross-flow direction). The base model for modifications [1] in dimensionless form is as follows:

$$
\begin{aligned}
& \ddot{X}+2 \zeta \dot{X}+\omega_{s t}^{2} X=\frac{a}{2 \pi S t} \Omega_{R}^{2}+\frac{b}{4 \pi S t} \Omega_{R}^{2} w-2 a \Omega_{R} \dot{X}+ \\
& +\frac{c}{2} \Omega_{R} q \dot{Y}+a \pi S t \dot{Y}^{2}+2 a \pi S t \dot{X}^{2}-b \Omega_{R} w \dot{X} \\
& \ddot{Y}+2 \zeta \dot{Y}+\omega_{s t}^{2} Y=\frac{c \Omega_{R}^{2}}{4 \pi S t} q-a \Omega_{R} \dot{Y}+2 a \pi S t \dot{X} \dot{Y}- \\
& \quad-\frac{b}{2} w \dot{Y} \Omega_{R}-c q \dot{X} \Omega_{R} ; \\
& \ddot{w}+f_{\text {damp } X}+4 \Omega_{R}^{2} w=A_{x} \ddot{X} ; \\
& \ddot{q}+f_{\text {damp } Y}+\Omega_{R}^{2} q=A_{y} \ddot{Y},
\end{aligned}
$$

where

$$
\begin{aligned}
& a=\frac{C_{D 0} \rho_{f} D^{2}}{4 \pi m_{*} S t} ; \quad b=\frac{C_{D 0}^{f l} \rho_{f} D^{2}}{4 \pi m_{*} S t} ; \quad c=\frac{C_{L 0} \rho_{f} D^{2}}{4 \pi m_{*} S t} ; \\
& f_{\text {damp } X}=2 \varepsilon_{x} \Omega_{R}\left(w^{2}-1\right) \dot{w} \\
& f_{\text {damp } Y}=\varepsilon_{y} \Omega_{R}\left(q^{2}-1\right) \dot{q} .
\end{aligned}
$$

Here, $X, Y$ are dimensionless structural displacements in in-line and cross-flow directions respectively, defined as $X=\frac{x}{D}$ and $Y=\frac{y}{D}$, where $x$ and $y$ are dimensional structural displacements, and $D$ is diameter of the structure; $w, q$ are fluctuating parts of drag and lift forces as wake coefficients; $\omega_{s t}$ is natural frequency of the structure; $\Omega_{R}$ is vortex shedding frequency; $S t$ is Strouhal number; $\zeta$ is damping ratio; $a, b, c$ are dimensionless complexes; $C_{D 0}$ is initial drag coefficient; $C_{D 0}^{f l}$ is initial fluctuating drag coefficient; $C_{L 0}$ is initial lift coefficient; $m_{*}$ is mass per unit length which contains mass of the structure per unit length and the fluid added mass per unit length; $\rho_{f}$ is fluid density; $A_{x}, A_{y}$ are empirical coupling coefficients connecting wake oscillators with equations of structural motion; $f_{\text {damp } X}$ and $f_{\text {dampY }}$ are nonlinear damping terms of fluid equations; $\varepsilon_{x}, \varepsilon_{y}$ are damping coefficients (Van der Pol parameters).

Mass ratio $\mu$ is defined in this research as suggested in [2] as relationship of the structural mass per unit length $m_{s}$ and the mass of displaced fluid per unit length $m_{f}$ :

$$
\mu=\frac{m_{s}}{m_{f}}=\frac{4 m_{s}}{\pi \rho_{f} D^{2}} .
$$

In this study, the accuracy of prediction with the Van der Pol damping terms (see Eq.(6)), applied in the model [1] for in-line and cross-flow wake equations, is compared with the accuracy provided by possible alternative fluid nonlinearities, as proposed in [5]:

- Modified Van der Pol terms $f_{\text {dampX }}=2 \varepsilon_{x 1} \Omega_{R} \dot{w} w^{2}-$ $2 \varepsilon_{x 2} \Omega_{R} \dot{w}$ and $f_{\text {damp } Y}=\varepsilon_{y 1} \Omega_{R} \dot{q} q^{2}-\varepsilon_{y 2} \Omega_{R} \dot{q}$;

- Modified Rayleigh terms $f_{\text {dampX }}=-2 \varepsilon_{x 1} \Omega_{R} \dot{w}+2 \frac{\varepsilon_{x 2}}{\Omega_{R}} \dot{w}^{3}$ and $f_{\text {damp } Y}=-\varepsilon_{y 1} \Omega_{R} \dot{q}+\frac{\varepsilon_{y 2}}{\Omega_{R}} \dot{q}^{3}$;

- Krenk-Nielsen terms $f_{\text {dampX }}=-2 \varepsilon_{x 1} \Omega_{R} \dot{w}+$ $2 \varepsilon_{x 2} \Omega_{R} \dot{w} w^{2}+2 \frac{\varepsilon_{x 3}}{\Omega_{R}} \dot{w}^{3}$ and $f_{\text {damp } Y}=-\varepsilon_{y 1} \Omega_{R} \dot{q}+$ $\varepsilon_{y 2} \Omega_{R} \dot{q} q^{2}+\frac{\varepsilon_{y 3}}{\Omega_{R}} \dot{q}^{3}$ suggested by Krenk and Nielsen [7];

- and Landl terms $f_{\text {dampX }}=2 \Omega_{R} \dot{w}\left(\varepsilon_{x 1}-\varepsilon_{x 2} w^{2}+\varepsilon_{x 3} w^{4}\right)$ and $f_{\text {damp } Y}=\Omega_{R} \dot{q}\left(\varepsilon_{y 1}-\varepsilon_{y 2} q^{2}+\varepsilon_{y 3} q^{4}\right)$ based on the work by Landl [6].

The alternative models developed using these terms are calibrated with the experimental data [2] in the next section. 
Table 1. Versions of the model calibrated for mass ratio 6.54 [2]

\begin{tabular}{|c|c|}
\hline Modification & Set of coefficients \\
\hline \multicolumn{2}{|c|}{ Prediction for both in-line and cross-flow displacement } \\
\hline Rayleigh - Van der Pol & $\begin{array}{l}C_{L 0}=1.83, C_{D 0}=1.73, C_{D 0}^{f l}=0.30, \varepsilon_{x}=1.8598, \varepsilon_{y}=0.3614, A_{x}=12.33, \\
A_{y}=2.50, C_{A}=0.67, K=1.09\end{array}$ \\
\hline \multicolumn{2}{|r|}{ Prediction for in-line displacement only } \\
\hline $\begin{array}{l}\text { Modified Rayleigh - } \\
\text { Modified Van der Pol }\end{array}$ & $\begin{array}{l}C_{L 0}=1.17, C_{D 0}=1.18, C_{D 0}^{f l}=0.64, \varepsilon_{x 1}=1.8976, \varepsilon_{x 2}=0.5211, \varepsilon_{y 1}=1.6508 \\
\varepsilon_{y 2}=1.0386, A_{x}=12.56, A_{y}=3.12, C_{A}=0.34, K=3.90\end{array}$ \\
\hline $\begin{array}{l}\text { Modified Rayleigh - } \\
\text { Modified Rayleigh }\end{array}$ & $\begin{array}{l}C_{L 0}=0.21, C_{D 0}=1.46, C_{D 0}^{f l}=0.87, \varepsilon_{x 1}=1.9601, \varepsilon_{x 2}=0.5841, \varepsilon_{y 1}=0.6688 \\
\varepsilon_{y 2}=1.3768, A_{x}=12.10, A_{y}=2.09, C_{A}=1.04, K=3.61\end{array}$ \\
\hline $\begin{array}{l}\text { Modified Van der Pol - } \\
\text { Krenk-Nielsen }\end{array}$ & $\begin{array}{l}C_{L 0}=1.14, C_{D 0}=1.62, C_{D 0}^{f l}=0.19, \varepsilon_{x 1}=0.0242, \varepsilon_{x 2}=0.5452, \varepsilon_{y 1}=1.9493 \\
\varepsilon_{y 2}=0.0214, \varepsilon_{y 3}=0.2491, A_{x}=1.02, A_{y}=3.2, C_{A}=1.96, K=0.17\end{array}$ \\
\hline Landl - Modified Rayleigh & $\begin{array}{l}C_{L 0}=0.26, C_{D 0}=0.58, C_{D 0}^{f l}=0.64, \varepsilon_{x 1}=0.8290, \varepsilon_{x 2}=0.8493, \varepsilon_{x 3}=0.8411 \\
\varepsilon_{y 1}=1.4904, \varepsilon_{y 2}=0.0192, A_{x}=5.05, A_{y}=5.02, C_{A}=1.00, K=1.09\end{array}$ \\
\hline \multicolumn{2}{|r|}{ Prediction for cross-flow displacement only } \\
\hline Landl - Van der Pol & $\begin{array}{l}C_{L 0}=0.38, C_{D 0}=0.37, C_{D 0}^{f l}=0.51, \varepsilon_{x 1}=0.8519, \varepsilon_{x 2}=0.7962, \varepsilon_{x 3}=0.9258 \\
\varepsilon_{y}=0.3479, A_{x}=6.36, A_{y}=4.49, C_{A}=1.03, K=1.80\end{array}$ \\
\hline Landl - Landl & $\begin{aligned} C_{L 0} & =1.03, C_{D 0}=0.59, C_{D 0}^{f l}=0.02, \varepsilon_{x 1}=0.3058, \varepsilon_{x 2}=0.5479, \varepsilon_{x 3}=0.0558 \\
\varepsilon_{y 1} & =0.0009, \varepsilon_{y 2}=0.1228, \varepsilon_{y 3}=0.3494, A_{x}=4.72, A_{y}=1.62, C_{A}=1.97, K=1.7\end{aligned}$ \\
\hline Landl - Modified Van der Pol & $\begin{aligned} C_{L 0} & =0.33, C_{D 0}=0.31, C_{D 0}^{f l}=0.47, \varepsilon_{x 1}=0.7922, \varepsilon_{x 2}=0.8399, \varepsilon_{x 3}=0.8535, \\
\varepsilon_{y 1} & =0.5322, \varepsilon_{y 2}=0.6653, A_{x}=9.81, A_{y}=5.77, C_{A}=1.19, K=1.51\end{aligned}$ \\
\hline Landl - Krenk-Nielsen & $\begin{aligned} C_{L 0} & =0.63, C_{D 0}=0.51, C_{D 0}^{f l}=0.34, \varepsilon_{x 1}=1.2909, \varepsilon_{x 2}=1.4159, \varepsilon_{x 3}=1.0042, \\
\varepsilon_{y 1} & =0.2581, \varepsilon_{y 2}=0.2273, \varepsilon_{y 3}=0.2260, A_{x}=5.39, A_{y}=4.38, C_{A}=1.00, K=1.59\end{aligned}$ \\
\hline
\end{tabular}

(a)

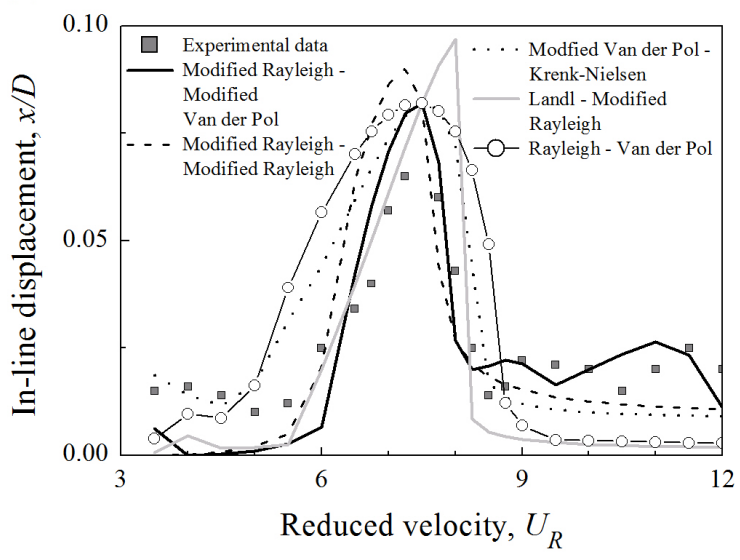

(b)

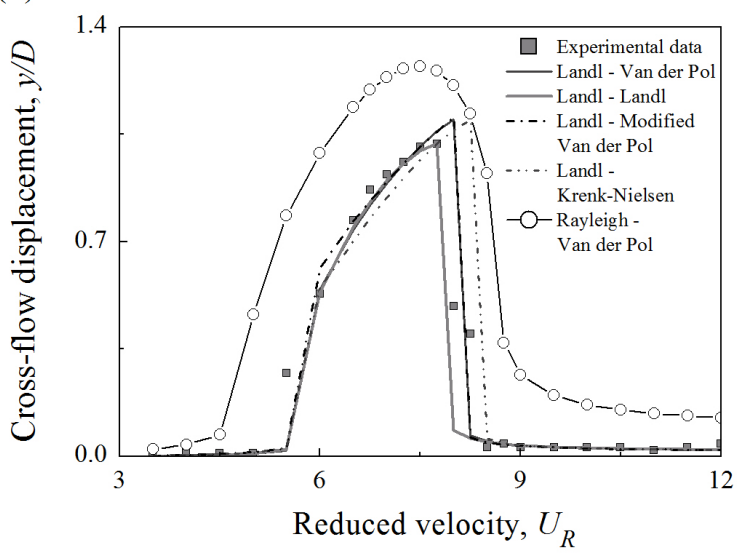

Figure 2. Displacement amplitudes generated by the model versions in comparison with experimental data [2]: a) calibrated inline responses of considered models for mass ratio 6.54 ; b) generated cross-flow response of the Rayleigh - Van der Pol system in comparison with calibrated cross-flow responses of other models for mass ratio 6.54.

\section{Calibration results}

Calibration of the dimensionless coefficients for the aformentioned wake oscillator models is performed following the algorithm in [5]. The main priority during calibration is given to the highest displacement amplitudes developed during the resonance. Experimental data [2] are for mass ratio 6.54. The highest displacement amplitudes here are approximately 0.082 for in-line direction and 1.02 for cross-flow direction. The highest amplitudes correspond to the reduced velocity 7.5 and 7.75 respectively.

The models are calibrated in respect to either in-line or cross-flow data, and the standard deviation is applied as the statistics of the signal. Eleven control points of experimental data [2] in either in-line or cross-flow direction are utilized to estimate the error of the model prediction. Calibration is conducted using the constrained nonlinear minimization tool in Matlab as detailed in [5].

The calibrated sets for medium mass ratio are presented in Table 1 in three groups. The first part of Table 1 is the combination of Rayleigh - Van der Pol dampings which allows relatively suitable prediction for both in-line and cross-flow displacement amplitudes at the same time as illustrated in Fig. 2. In this case, Fig. 2a demonstrates the calibration result for in-line direction, and Fig. 2b, Figs 3 and 4 show the results of validation on the same exper- 
(a)

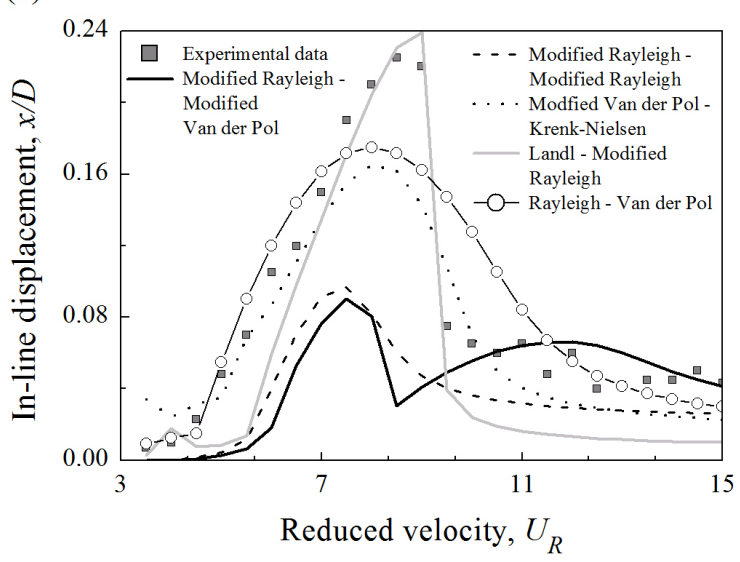

(c)

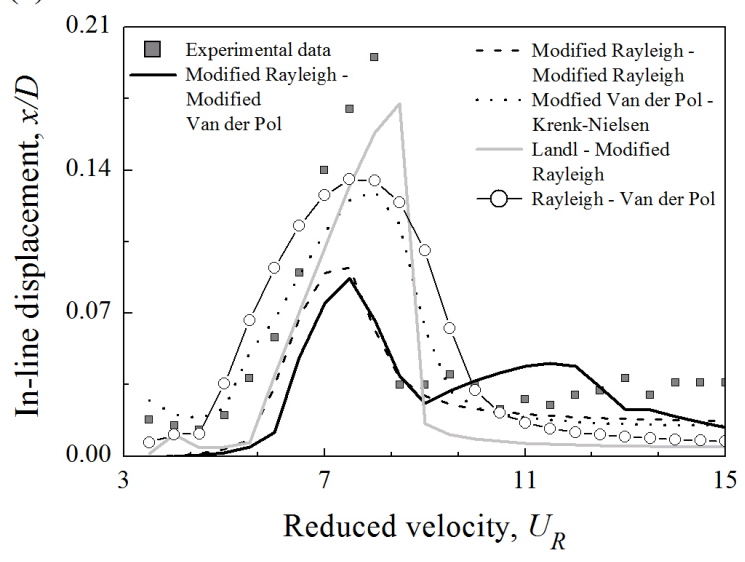

(e)

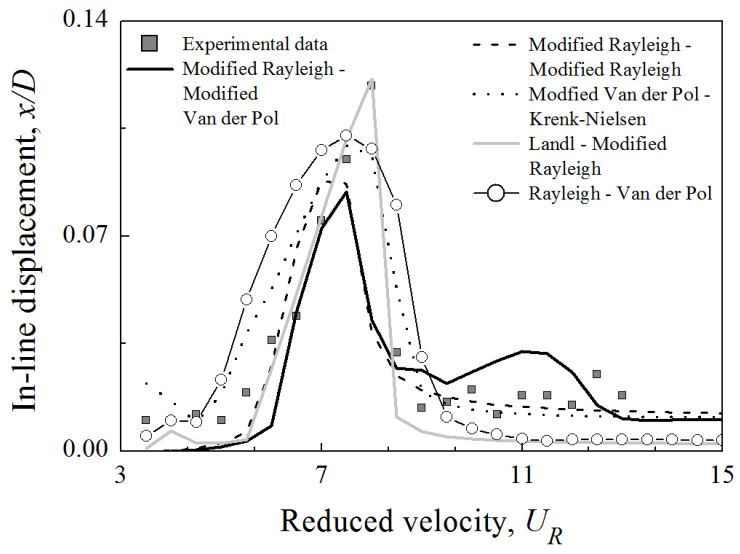

(b)

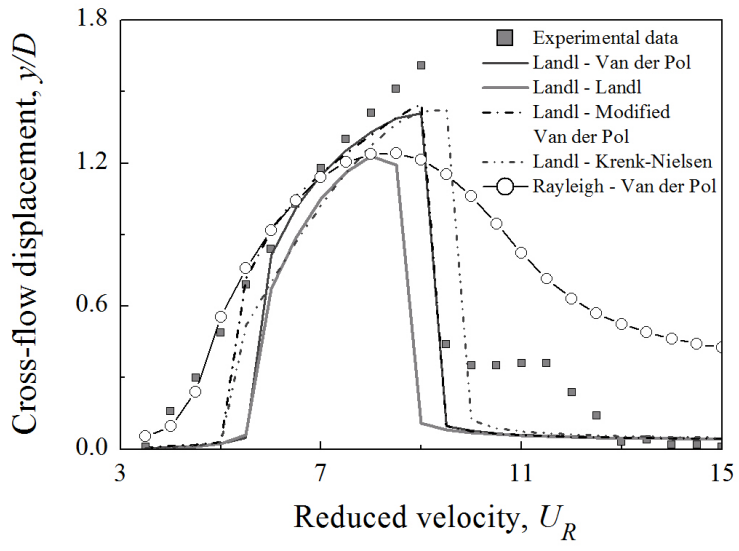

(d)

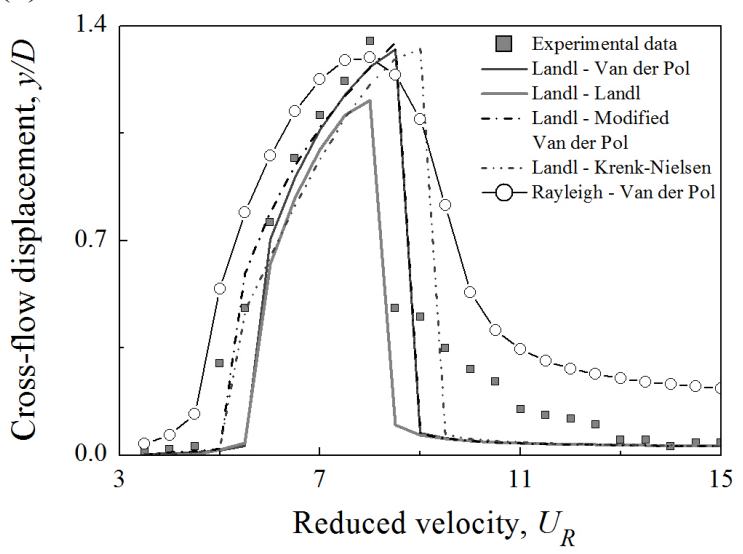

(f)

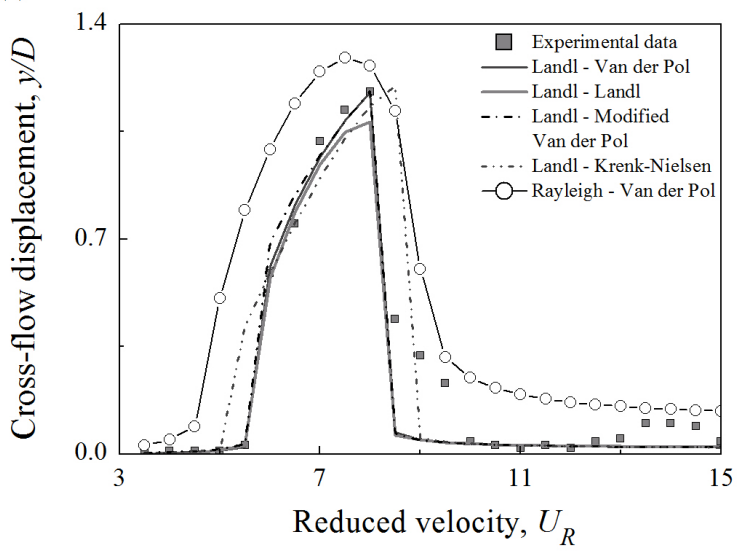

Figure 3. Displacement amplitudes generated by the model versions in comparison with experimental data [2]: a) in-line response for mass ratio 2.36; b) cross-flow response for mass ratio 2.36; c) in-line response for mass ratio 3.68; d) cross-flow response for mass ratio 3.68; e) in-line response for mass ratio 5.19; f) cross-flow response for mass ratio 5.19.

imental set-up [2]. Hence, Figs 2a, 3a, 3c, 3e, 4a, and $4 \mathrm{c}$ show the comparison of fit by the models calibrated with in-line displacement record for mass ratio 6.54; and Figs $2 \mathrm{~b}, 3 \mathrm{~b}, 3 \mathrm{~d}, 3 \mathrm{f}, 4 \mathrm{~b}$, and $4 \mathrm{~d}$ provide the comparison of the combination of Rayleigh - Van der Pol dampings (calibrated with the in-line displacement record) with the options calibrated with cross-flow displacement record for mass ratio 6.54 .

The second part of Table 1 present options of the fluid nonlinearities which are able to predict in-line displacement only when calibrated with mass ratio 6.54 [2], and they are shown in Figs 2a, 3a, 3c, 3e, 4a, and 4c for the range of mass ratios. Those are options of Modified 
(a)

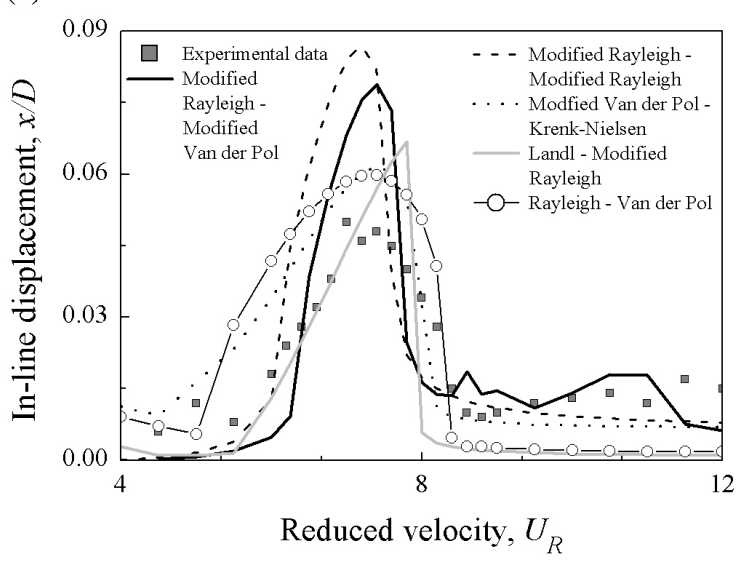

(c)

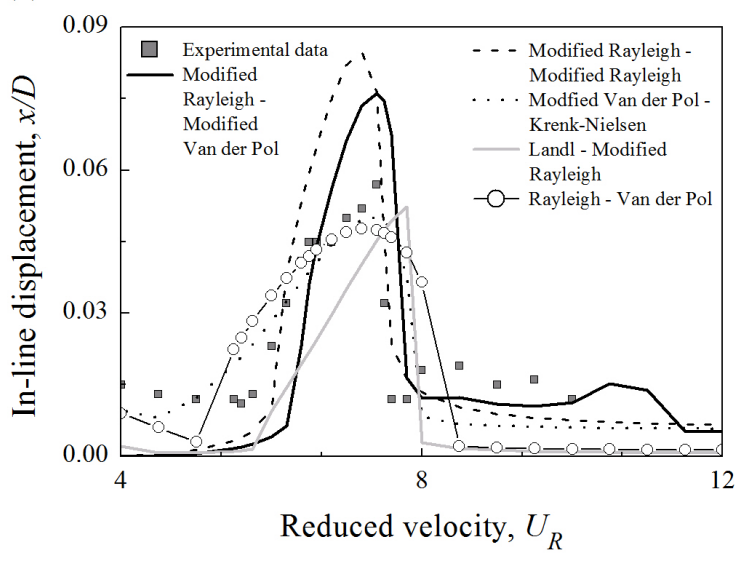

(b)

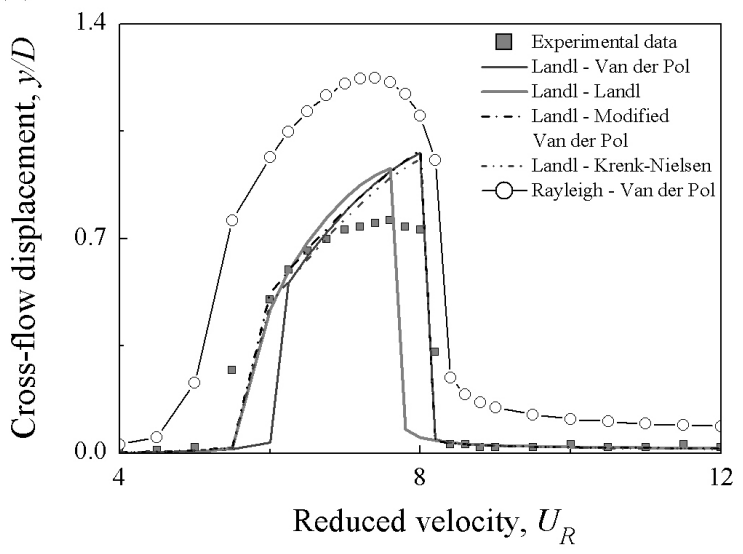

(d)

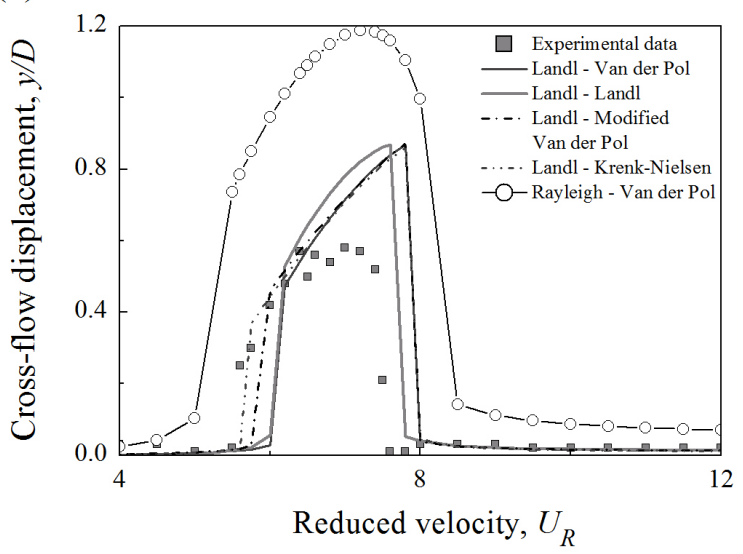

Figure 4. Displacement amplitudes generated by the model versions in comparison with experimental data [2]: a) in-line response for mass ratio 8.76; b) cross-flow response for mass ratio 8.76; c) in-line response for mass ratio 10.63; d) cross-flow response for mass ratio 10.63 .

Rayleigh - Modified Van der Pol, Modified Rayleigh Modified Rayleigh, Modified Van der Pol - Krenk-Nielsen, and Landl - Modified Rayleigh fluid nonlinearities. Fig. 2a demonstrates the result of calibration with the in-line displacement record for mass ratio 6.54, and Figs 3a, 3c, 3e, $4 \mathrm{a}, 4 \mathrm{c}$ are the results of validation on the same experimental set-up [2].

The third block of Table 1 contains the fluid nonlinearities to forecast cross-flow displacement only when calibrated with mass ratio 6.54 [2]: Landl - Van der Pol, Landl - Landl, Landl - Modified Van der Pol, and Landl - KrenkNielsen fluid nonlinearities. Accuracy of their fit is illustrated in Figs 2b, 3b, 3d, 3f, 4b, and 4d for the range of mass ratios. Fig. $2 b$ is the result of calibration with mass ratio 6.54; and Figs 3b, 3d, 3f, 4b, and $4 d$ are the results of validation on the same experimental set-up [2].

\section{Discussion}

Fig. 2a demonstrates the advantageous fit provided by fluid nonlinearities Modified Rayleigh - Modified Van der Pol calibrated with the in-line displacement amplitudes at mass ratio 6.54 [2]. It manifests in the correct position of the resonance peak, the correct highest displacement amplitude, and the best representation of the lower branch among considered options. Figs 3a, 3c, 3e, 4a, and 4c, however, illustrate that the best quality of the fit (in-line peak height and width) throughout the range of mass ratio from 2.0 to 9.0 belongs to the combination of Landl Modified Rayleigh dampings.

Figs $2 \mathrm{a}, 3 \mathrm{e}$ and $4 \mathrm{a}$ prove that the range of the correct predictions by options with Modified Rayleigh - Modified Van der Pol and Modified Rayleigh - Modified Rayleigh dampings is very narrow - around mass ratio 6.0-7.0. The combination of nonlinearities Modified Rayleigh - Modified Van der Pol can be seen as a more interesting due to providing relatively safe versions of lower branch at different mass ratios.

At the same time, options with Rayleigh - Van der Pol and Modified Van der Pol - Krenk-Nielsen dampings, as appears in Figs 2a, 3e, 4a, and 4c, provide a suitable, safe fit for in-line displacement amplitudes over the mass ratio range approximately from 6.0 to 10.0 . 
Fig. 2b shows very accurate predictions by Landl Van der Pol, Landl - Landl, Landl - Modified Van der Pol dampings of the cross-flow peak at mass ratio 6.54. Variation of mass ratio in Figs 2b, 3b, 3d, 3f, 4b, and 4d reveals the advantageous prediction by Landl - Van der Pol, Landl - Modified Van der Pol, and also Landl - Krenk-Nielsen dampings from approximately mass ratio 3.7 to mass ratio 9.0. Rayleigh - Van der Pol damping (from the first part of Table 1) significantly overestimates the cross-flow displacement amplitudes when mass ratio exceeds 6.0. Landl - Landl fluid nonlinearities with identified set of coefficients tend to predict cross-flow peak to be narrower than it appears in experiments [2].

\section{Conclusions}

The considered modifications of the base model [1] show that predicted in-line and cross-flow displacements are more accurate if modelled separately using different damping types than using only one version of the model, as follows from the analysis of Figs 2, 3 and 4.

Option of Landl - Modified Rayleigh damping calibrated with the in-line displacement amplitudes allows to predict in-line displacement in the largest range of mass ratio: approximately from 2.0 to 9.0, as illustrated in Figs 2a, 3a, 3c, 3e, 4a, and 4c.

Options of Landl - Van der Pol, Landl - Modified Van der Pol, Landl - Krenk-Nielsen dampings calibrated with cross-flow displacement amplitudes are proved reliable to predict cross-flow displacement only in the range of mass ratio from approximately 3.7 until 9.0. It is substantiated by Figs 2b, 3b, 3d, 3f, 4b, and 4d.

Option of Rayleigh - Van der Pol damping calibrated with the in-line displacement amplitudes predicts in-line displacement safe and relatively correctly for the range of mass ratio from approximately 6.0 to 10.0 , and cross-flow displacement - from 3.7 to 6.0, as revealed by Figs 2, 3 and 4.

Analysis of Table 1 allows to suggest that Landl damping in in-line fluid equation enables relatively accurate prediction of cross-flow displacement amplitudes. This makes the combination of Landl - Modified Rayleigh fluid nonlinearities the most perspective option for the future development. Hence, the next stages of this research should focus on the synthetic oscillators specifically designed for the models of 2 degrees-of-freedom structures, and definitely involve more experimental data for calibration and validation.

\section{Acknowledgements}

V.K. would like to acknowledge the support of the Industrial University of Tyumen, Tyumen, Russia, and the State Program "Global Education", Russia.

This work was performed using the Maxwell High Performance Computing Cluster funded by the University of Aberdeen.

Authors also would like to express their gratitude to Dr. Andrew Starkey for advices regarding optimization procedures and to Naveed Khan for technical advices on accelerating computations.

\section{References}

[1] Postnikov A., Pavlovskaia E., Wiercigroch M. 2DOF CFD calibrated wake oscillator model to investigate vortex-induced vibrations, International Journal of Mechanical Sciences 127, 176-190 (2017).

[2] Stappenbelt B., Lalji F. Vortex-induced vibration super-upper response branch boundaries, International Journal of Offshore and Polar Engineering 2(18), 99105 (2008).

[3] Jauvtis N., Williamson C.H.K. The effect of two degrees-of-freedom on vortex-induced vibration at low mass and damping, Journal of Fluid Mechanics 509, 23-62 (2008).

[4] Kurushina V., Pavlovskaia E. Wake oscillator equations in modelling vortex-induced vibrations at low mass ratio, OCEANS 2017 - Aberdeen, 1-6 (2017). IEEE. DOI: 10.1109/OCEANSE.2017.8084644

[5] Kurushina V., Pavlovskaia E., Postnikov A., Wiercigroch M. Calibration and comparison of VIV wake oscillator models for low mass ratio structures. Submitted for publication.

[6] Landl R. A mathematical model for vortex-excited vibrations of bluff bodies, Journal of Sound and Vibration 2(42), 219-234 (1975).

[7] Krenk S., Nielsen S.R.K. Energy balanced double oscillator model for vortex-induced vibrations, Journal of Engineering Mechanics 3(125), 263-271 (1999). 